\title{
GE23
}

\section{Examining Three New Methods to Validate Re- fracture Models in Unconventional Reservoirs}

\author{
H. Shaikh* (E-Tech Consulting Services), T. Hasan (University of Calgary), \\ O. Ogunrewo (Global Ace Solutions), A. Esquivel (Independent \\ Researcher) \& N. Malik (Independent Researcher)
}

\section{SUMMARY}

As cost effectiveness becomes important in producing hydrocarbons, some operators are returning to their mature field to re-fracture the reservoir in order to enhance the overall recovery. Re-fracturing has been performed on tight reservoirs on multiple wells, but little has been done to validate the results from the treatment. This paper will investigate three different approaches to validate the results from re-fracturing unconventional reservoirs and make recommendations for re-stimulation of wells in order to improved the economics in a low commodity price market. 


\section{Introduction}

Tight oil and gas reservoirs typically need to be hydraulically fractured in order to extract the hydrocarbons (Liu et al. 2008; Cherian et al. 2015). Rigorous characterization of rock properties is essential to designing effective hydraulic fracture treatments (Hamza et al. 2015; Hamza, Gu, and Quirein 2016; Narasimhan et al. 2016). As cost effectiveness becomes important in producing hydrocarbons, some operators are returning to their mature field to re-fracture the reservoir in order to enhance the overall recovery. Re-fracturing has been performed on tight reservoirs on multiple wells (Wright et al. 1994), but little has been done to validate the results from the treatment. This study will investigate three different approaches to validate the results from re-fracturing unconventional reservoirs.

\section{Method and/or Theory}

Previous authors have described multi-domain workflows for field development studies (Narasimhan et al. 2015; Cherian et al. 2015). Geomechanical data was coupled with Petrophysical analysis to build a 3D fracture model using which fracture pressure history matching was performed on a representative well in the area of interest. Production history matching was performed to validate the reservoir simulation model. Geomechanical properties were recomputed post-production to characterize the effects of depletion on rock mechanical properties using the assumption of only pore pressure depletion, linear depletion, and non-linear depletion. These three methods were then used to reconstruct the fracture model to simulate re-stimulation of the reservoir using different pumping treatments.

\section{Examples}

This section shows the stress field post-depletion for three scenarios:

- Pore pressure depletion as shown in Figure 1

- Linear depletion as shown in Figure 2

- Non-linear depletion as shown in Figure 3

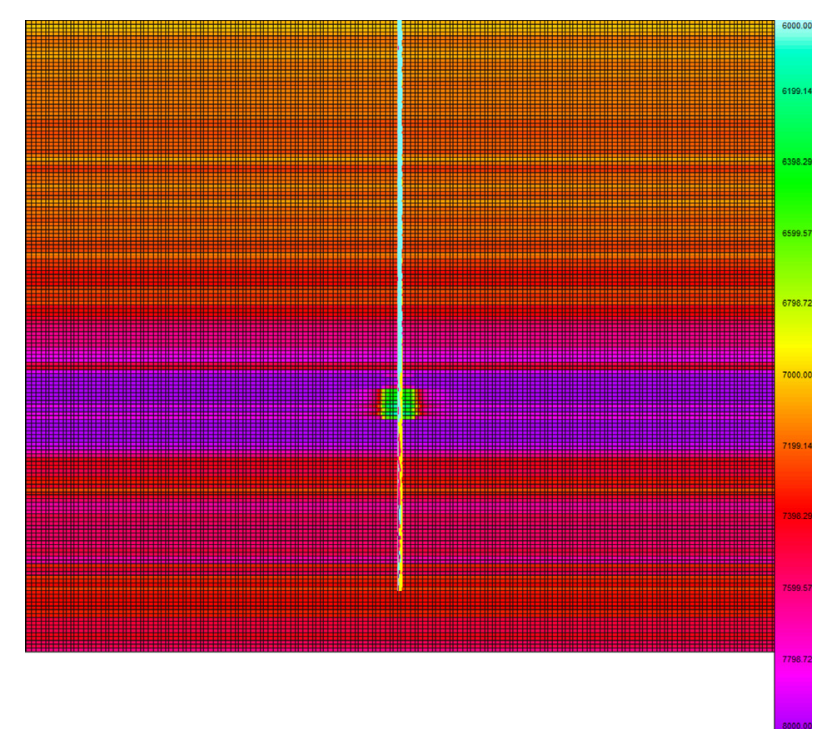

Figure 1 Stress field surrounding a well post-depletion using the pore pressure depletion model. 


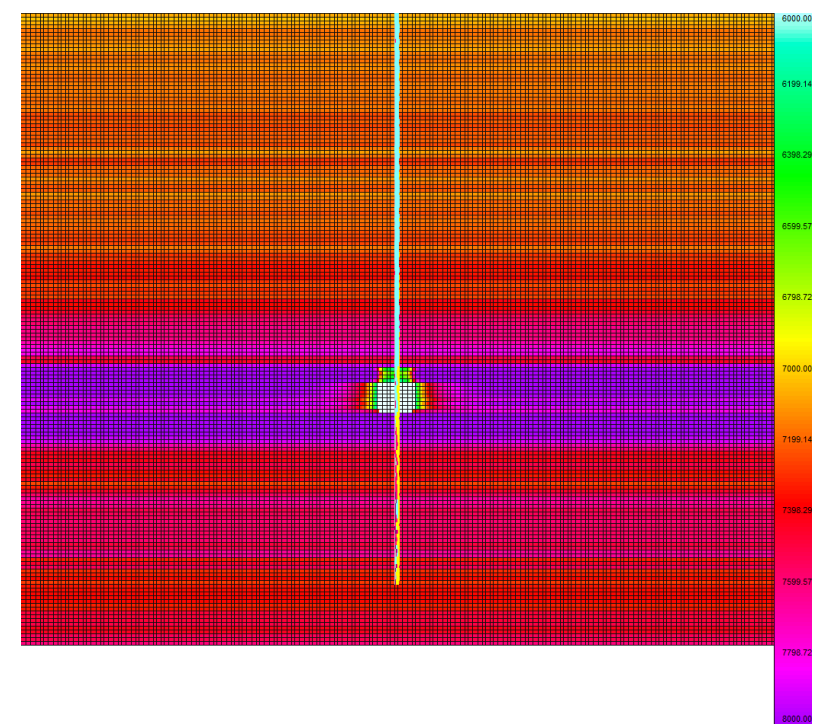

Figure 2 Stress field surrounding a well post-depletion using the linear depletion model.

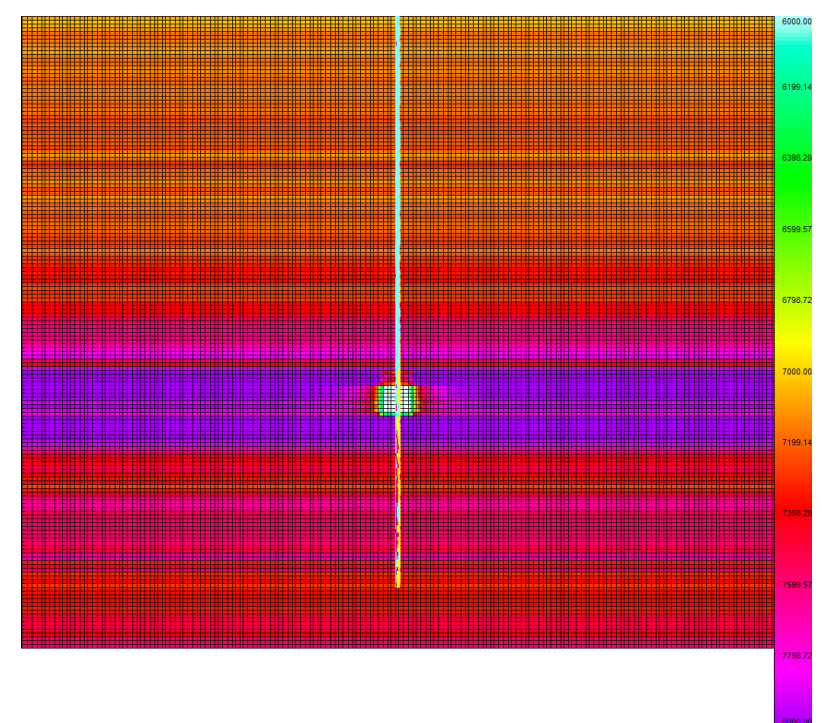

Figure 3 Stress field surrounding a wellbore using non-linear depletion model. 


\section{Examples}

This section shows fracture geometries using 'Plug and Perf' and 'Sliding Sleeve' completions for refracture treatments for the three abovementioned scenarios in Figure 4 and Figure 5.

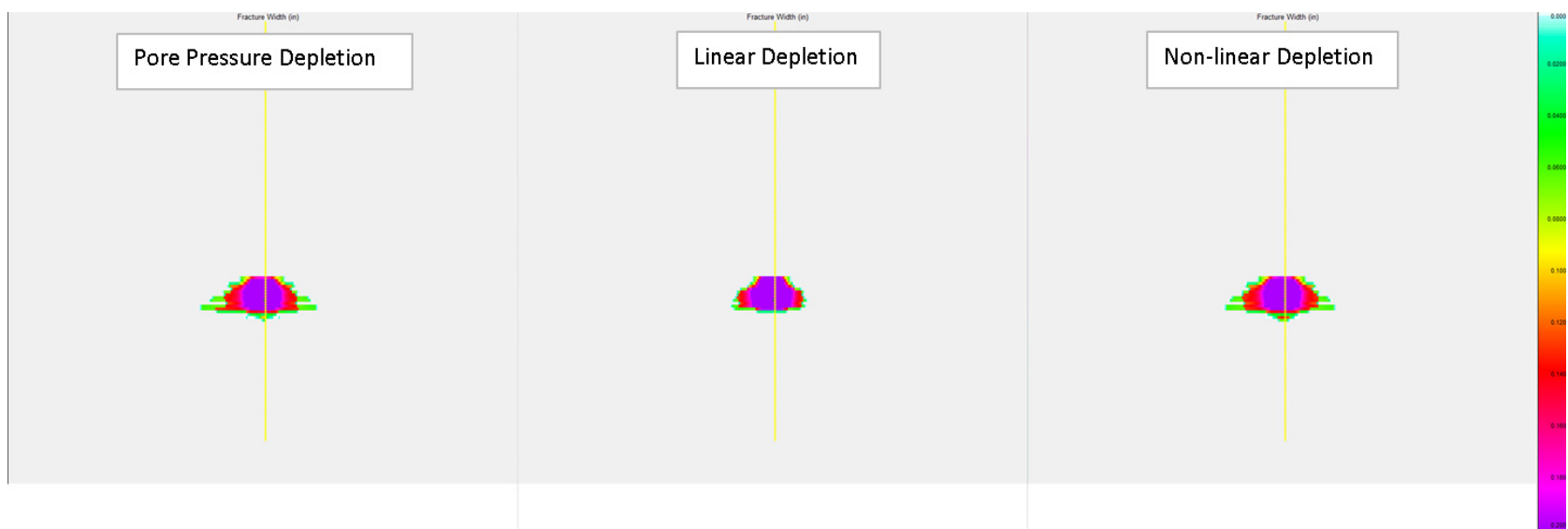

Figure 4 Fracture geometries using a 'Plug and Perf' designs.

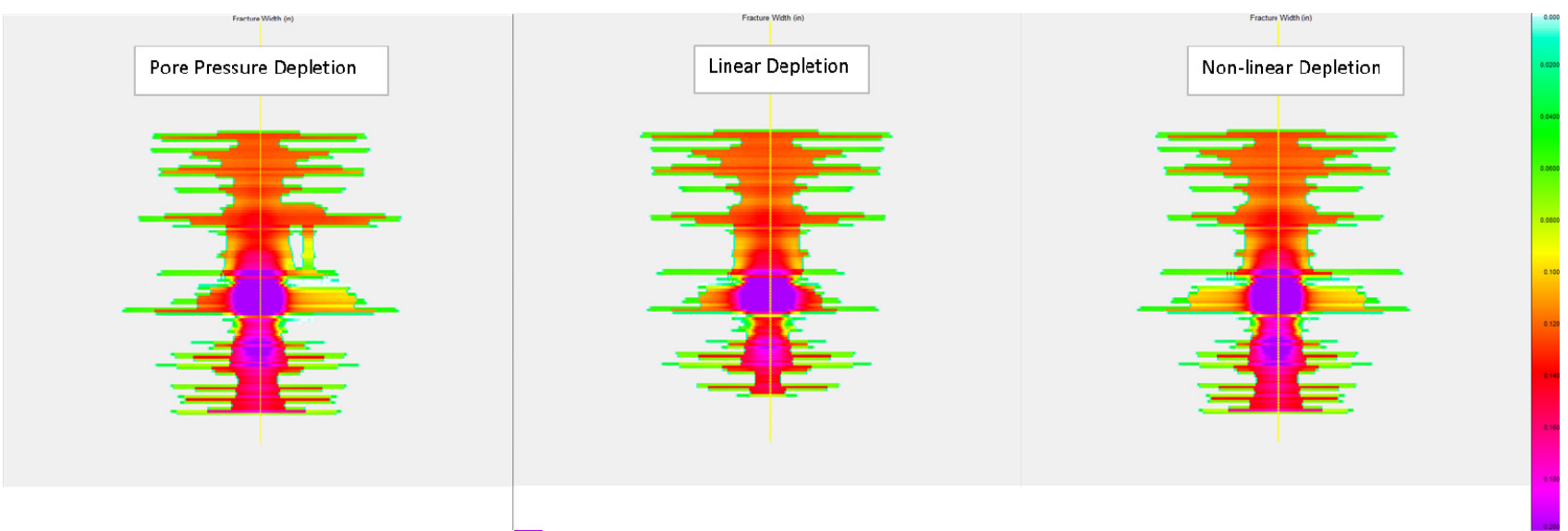

Figure 5 Fracture geometries using a 'Sliding Sleeve' design.

Depending on the volume of fluid pumped, the geometries obtained from the re-fracture simulations are dependent on the re-computation of Geomechanical properties post-depletion. These properties were validated using laboratory results. The geometry evolution with time indicated consistent geometries using the three methods when typical Plug and Perf volumes were pumped. Differences in geometries were observed only after a very large volume of fluid suggesting the use of the rigorous non-linear depletion model (third method).

\section{Conclusions}

Validated models are important when designing re-fracture treatments to avoid the risk of incorrect proppant placement or undesirable bashing of an offset well. Good understanding of re-fracture models helps operators make informed decisions related to their completion design and hence improving the overall hydrocarbon recovery from the field.

Modeling of re-fracture treatments has been very challenging for the industry professionals as little has been done to validate the results. This study describes the guidelines to follow when modeling refracture treatments with results validated from field and laboratory testing. Unconventional reservoirs hold good potential for re-fracturing if the treatments are designed properly. 


\section{References}

Cherian, Bilu V., Matthew McCleary, Samuel Fluckiger, Nathan Nieswiadomy, Brent Bundy, Sarah Edwards, Rafif Rifia, et al. [2015] Production Performance in the In-Fill Development of Unconventional Resources. SPE/CSUR Unconventional Resources Conference. Society of Petroleum Engineers. doi:10.2118/175963-MS.

Hamza, Farrukh, Cheng Chen, Ming Gu, John Quirein, Vladimir Martysevich, and Luis Matzar. [2015] Characterization of Anisotropic Elastic Moduli and Stress for Unconventional Reservoirs Using Laboratory Static and Dynamic Geomechanical Data. SPE/CSUR Unconventional Resources Conference. Society of Petroleum Engineers. doi:10.2118/175907-MS.

Hamza, Farrukh, Ming Gu, and John Quirein. [2016] Improved Characterization of Anisotropic Elastic Moduli and Stress for Unconventional Reservoirs Using Laboratory Mineralogy, TOC, Static, and Dynamic Geomechanical Data. SPWLA Annual Logging Symposium. Society of Petrophysicists and Well Log Analysts.

Liu, He, Zhongxiao Lan, Guoliang Zhang, Feng Hou, Xiuqing He, and Xinghui Liu. [2008] Evaluation of Refracture Reorientation in Both Laboratory and Field Scales. SPE International Symposium and Exhibition on Formation Damage Control. Society of Petroleum Engineers. doi:10.2118/112445-MS.

Narasimhan, Santhosh, Matt McCleary, Samuel D Fluckiger, James Gray, Bilu V. Cherian, Hamza Shaikh, and Olubiyi Olaoye. [2015] Using the Wrong Method to Estimate Stress From Depletion Causes Significant Errors in Predicting Wellbore Integrity and Fracture Geometry. SPE Eastern Regional Meeting. Society of Petroleum Engineers. doi:10.2118/177310-MS.

Narasimhan, Santhosh, Hamza Shaikh, James K Gray, Bilu V Cherian, Olubiyi Olaoye, Rafif Rifai, Kristina Kublik, Matt McCleary, Samuel D. Fluckiger, and Munir Sharf-Aldin. [2016] Effect of Horizontal Stress Models and Biot Poro-Elasticity on Predicted Fracture Geometry. SPE Hydraulic Fracturing Technology Conference. Society of Petroleum Engineers. doi:10.2118/179162-MS.

Wright, C.A., R.A. Conant, D.W. Stewart, and P.M. Byerly. [1994] Reorientation of Propped Refracture Treatments. Rock Mechanics in Petroleum Engineering. Society of Petroleum Engineers. doi:10.2118/28078-MS. 